\title{
Discrete or diffuse intramedullary tumor? Contrast-enhanced intraoperative ultrasound in a case of intramedullary cervicothoracic hemangioblastomas mimicking a diffuse infiltrative glioma: technical note and case report
}

\author{
Ignazio G. Vetrano, MD, ${ }^{1,2}$ Francesco Prada, MD, ${ }^{1}$ llaria F. Nataloni, MD, ${ }^{2,3}$ \\ Massimiliano Del Bene, MD, ${ }^{1}$ Francesco DiMeco, MD, ${ }^{1,4}$ and Laura G. Valentini, MD' \\ 'Department of Neurosurgery, Fondazione IRCCS "Istituto Neurologico C. Besta"; ${ }^{2}$ University of Milan; and ${ }^{3}$ Department \\ of Thoracic Surgery, Fondazione IRCCS Cà Granda - Ospedale Maggiore Policlinico, Milan, Italy; and "Department of \\ Neurosurgery, Johns Hopkins University, Baltimore, Maryland
}

\begin{abstract}
Hemangioblastomas are benign, highly vascularized intramedullary lesions that may also extend into the intradural space. Surgery represents the standard therapy, with the goal of obtaining complete resection even at the risk of neurological morbidity. MRI is the gold standard for diagnosis and assessment of intramedullary tumors. Nevertheless, sometimes MRI may not accurately differentiate between different types of intramedullary tumors, in particular if they are associated with syringes or intra- and peritumoral cysts. This could subsequently affect surgical strategies. Intraoperative ultrasound (ioUS) has become in the last few years a very useful tool for use during neurosurgical procedures. Various ioUS modalities such as B-mode and Doppler have been applied during neurosurgical procedures. On the other hand, the use of contrast-enhanced ultrasound (CEUS) is not yet well defined and standardized in this field. We report a case of a young patient harboring a cervicothoracic intramedullary tumor, for which the preoperative neuroradiological diagnosis was in favor of a diffuse astrocytoma with nodular components whereas ioUS demonstrated 3 distinct intramedullary nodules. CEUS showed highly vascularized lesions, compatible with hemangioblastomas. These findings, particularly those obtained with CEUS, allowed better definition of the lesions for diagnosis, enhanced understanding of the physiopathological aspects, and permitted the localization of all 3 nodules, thus limiting spinal cord manipulation and allowing complete resection of the lesions, with an uneventful postoperative neurological course.

To the best of our knowledge, this is the first report of the use of intraoperative CEUS in a case of intramedullary hemangioblastoma.
\end{abstract}

http://thejns.org/doi/abs/10.3171/2015.5.FOCUS15162

KEY WORDS contrast-enhanced ultrasound; hemangioblastoma; intramedullary tumor; intraoperative ultrasound; spinal tumor

$\mathrm{H}$ EMANGIOBLASTOMAS are benign, highly vascularized tumors. They usually represent $2 \%$ to $15 \%$ of reported series of intramedullary spinal cord lesions. ${ }^{4,7,16,17,30,31}$ Progressive motor and sensitivity dysfunctions are major symptoms of cervical and dorsal tumors, while bowel and bladder signs are typically associated with lower thoracic or lumbar tumors. ${ }^{4-6,11,14,17,31}$ Spinal hemangioblastomas can be superficial, while some lie deeply embedded within the spinal cord. $., 6,11,17,18$
MRI is the gold standard for the diagnosis and assessment of intradural tumors. On MRI, hemangioblastomas appear as discrete nodular lesions, with homogeneous enhancement after Gd administration. . $^{1,3,6,10,19,27,32}$ Normal collateral findings are an edematous spinal cord with perilesional cyst or associated syrinx.

Nevertheless, MRI may not always differentiate accurately between different types of intramedullary tumors, in particular when they are associated with syringes or

ABBREVIATIONS CEUS = contrast-enhanced ultrasound; iCEUS = intraoperative CEUS; ioUS = intraoperative ultrasound; UCA = ultrasonographic contrast agent; US = ultrasound.

SUBMITTED March 31, 2015. ACCEPTED May 12, 2015.

INCLUDE WHEN CITING DOI: 10.3171/2015.5.FOCUS15162.

DISCLOSURE The authors report no conflict of interest concerning the materials or methods used in this study or the findings specified in this paper. 
intra- and peritumoral cysts. For this reason, the use of intraoperative imaging tools such as ultrasound (US) can be very helpful in formulating the surgical strategy. ${ }^{24}$

We report a case of a young patient harboring a cervicothoracic intramedullary tumor. In this patient, preoperative neuroradiological findings were in favor of a diffuse, infiltrative intramedullary lesion with multiple nodules. However, the use of intraoperative US (ioUS) and, in particular, of Doppler and contrast-enhanced US (CEUS) indicated instead that there were 3 distinct highly vascularized intramedullary cervical tumors. CEUS is a relatively new technique that has been applied in a number of ultrasonographic procedures related to the abdominal compartment and other organs. ${ }^{20,21}$ With the use of ultrasonographic contrast agents (UCAs), in our case it was possible to obtain direct real-time characterization of neoplastic lesions, specifically in regard to their vascularization. Intraoperative ultrasonographic findings that ultimately altered the surgical strategy in this case are reported in detail.

\section{Case Report}

History and Examination

A 14-year-old male patient was admitted to our institution in January 2015 with a long history of slowly progressive cervical and thoracic pain, usually arising during physical efforts. Neurological examination showed hyposthenia of the right arm, more severe distally, with hypoesthesia of the same region. Symmetric hyperreflexia in all 4 extremities was also noted.

MRI scans depicted diffuse alteration of the spinal cord at the C2-T2 level, which appeared enlarged, with an altered signal. Three nodular lesions were present at the T-4, T-1, and C-7 levels, with blurred margins, all surrounded by cystic cavities along the spinal axis. After $\mathrm{Gd}$ injection all 3 nodules showed intense enhancement, as well as cyst capsules and altered spinal cord between the nodules. The neuroradiological diagnosis was that of a diffuse intramedullary tumor, compatible with an astrocytoma (Fig. 1).

\section{Operation and Ultrasound Findings}

To achieve a partial debulking and to obtain specimens for histopatological diagnosis, the patient underwent surgery in the prone position, with the assistance of neurophysiological monitoring and US guidance. A posterior midline approach was performed. Laminae C-2 to T-4 and spinous processes were removed. To confirm the adequacy of the bone removal before dural opening, ioUS was performed, as it has become our practice during surgery for spinal tumor at our institution. The surgical field was examined with transdural insonation through the posterior laminotomy on both the axial and sagittal axes, using direct B-mode, Doppler, and CEUS. We used a last-generation US system (Esaote MyLab with Virtual Navigator) with a multifrequency $(3-11 \mathrm{MHz})$ linear probe.

The standard B-mode imaging examination showed an enlarged edematous spinal cord with multiple cystic cavities (Fig. 2). Three ovular, hyperechoic lesions, with cranial cystic areas and an ill-defined border caudally, were present. It was not possible to differentiate edema-

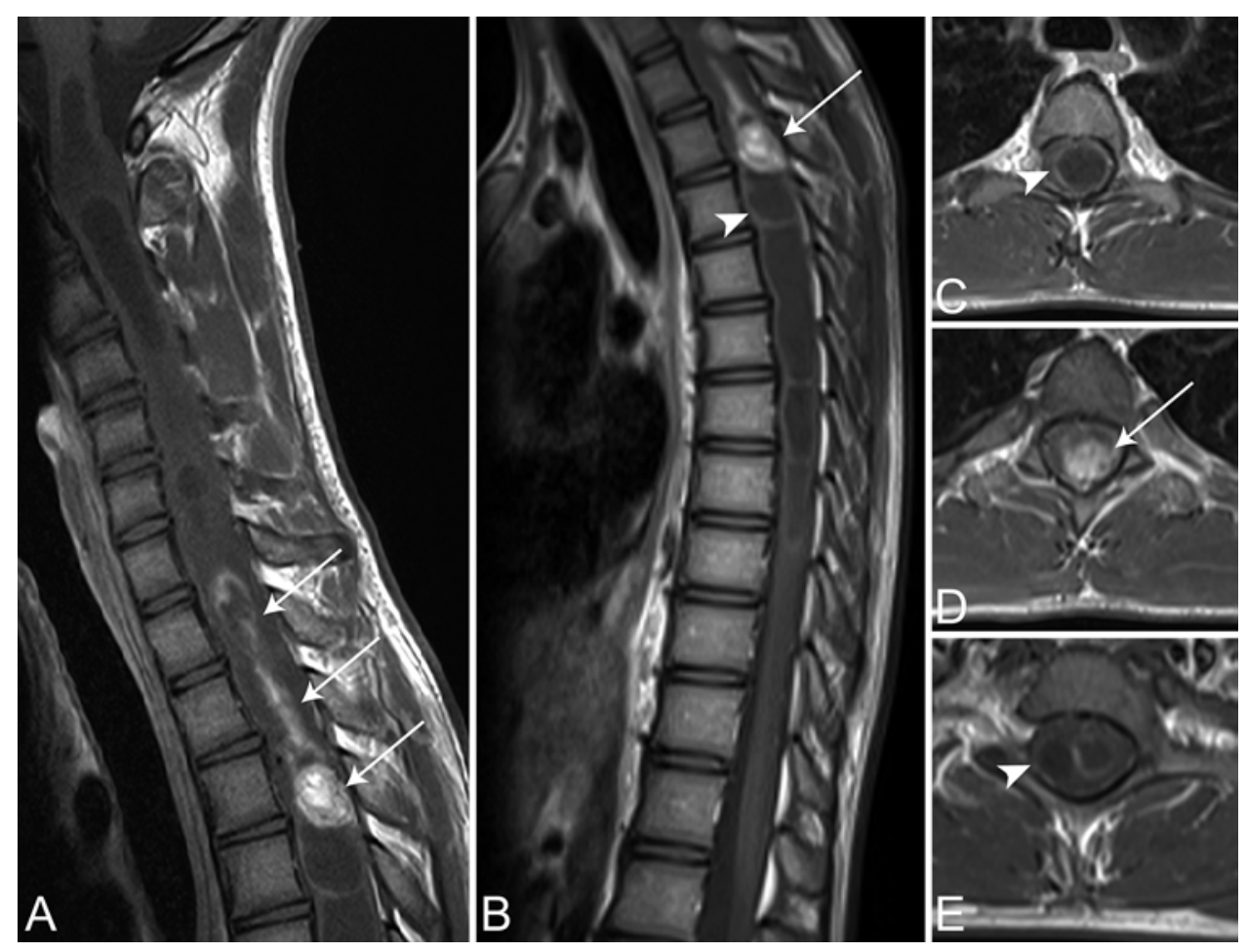

FIG. 1. Preoperative MR images. Sagittal T1 cervical (A), thoracic (B), and axial T1 (C-E) post-Gd images. Panels A and B show widening of the spinal cord at the C2-T4 level, with 3 nodular lesions (arrows). Cyst capsules and the altered spinal cord in between the nodules show enhancement after $\mathrm{Gd}$ in panels $\mathrm{C}$ and $\mathrm{E}$ (arrowheads). In panel $\mathrm{D}$ nodular contrast enhancement is also visible (arrows). 

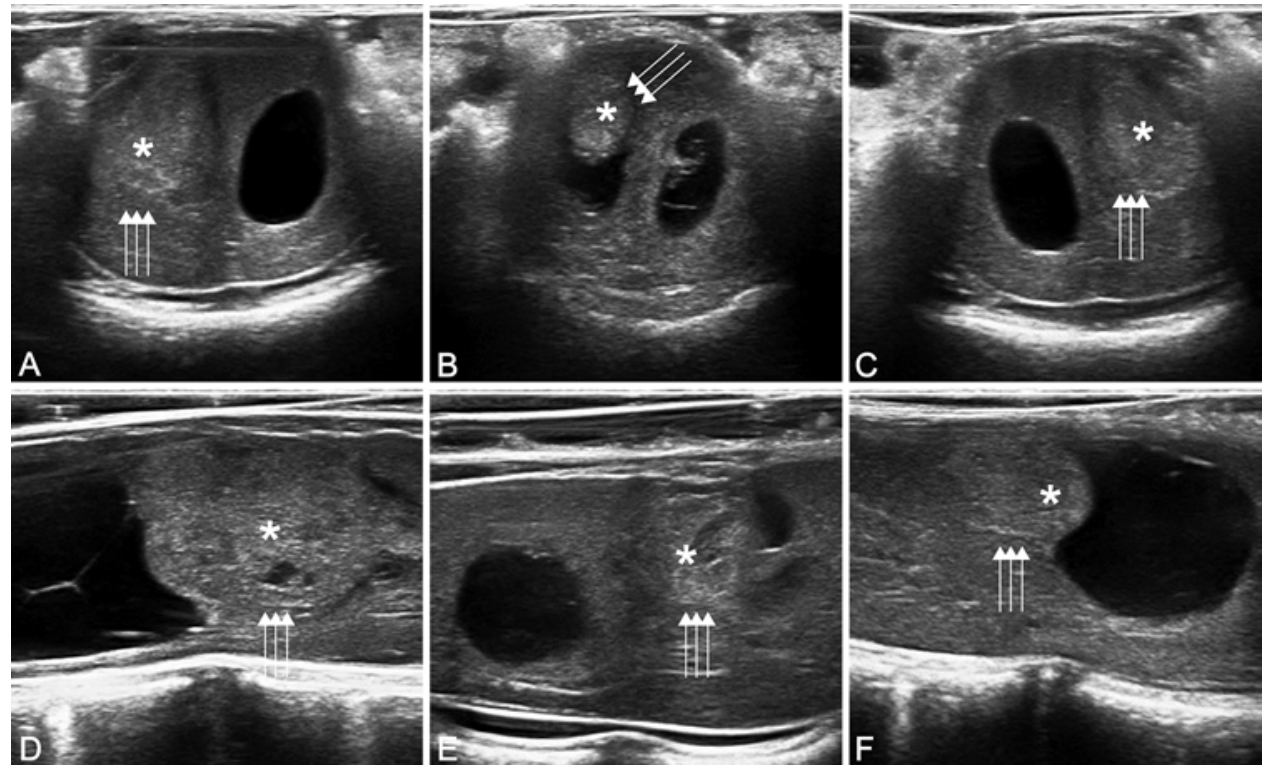

FIG. 2. Intraoperative B-mode images prior to resection. A-C: Axial scans of 3 nodular lesions (asterisks) surrounded by cystic cavities or syrinx. D-F: Corresponding sagittal B-mode scans. It is not possible to clearly differentiate edematous spinal cord (triple arrows) from infiltrative lesion.

tous spinal cord from an infiltrative lesion. The Eco-Color Doppler mode showed multiple high- and low-flow vessels within the lesion and enlarged draining veins.

After having scanned the lesion with navigated US in B-mode and with color Doppler, we then decided to also perform intraoperative CEUS (iCEUS), as previously described in other papers from our group. ${ }^{22,23}$ We followed the European Federation of Societies for Ultrasound in Medicine and Biology (EFSUMB) guidelines on CEUS, ${ }^{21}$ in an off-label setting.

The US probe was positioned at the level of the larger lesion in a sagittal plane and the main lesion was identified; then we switched to the specific contrast-tuned imaging algorithm. Sulfur hexafluoride-filled lipidic microbubbles (SonoVue), a second-generation purely intravascular UCA, was injected intravenously by the anesthesiologist, as a bolus of $2.4 \mathrm{~mL}(5 \mathrm{mg} / \mathrm{mL})$, followed by a flush of $10 \mathrm{ml}$ of saline solution. The UCA generally arrives in the observed field 30 seconds after injection, lasts in the bloodstream approximately 5 minutes, and is then excreted through the lungs and kidneys. The surgical field had been examined with iCEUS for approximately 3 minutes, and the subsequent harmonic signals due to UCA vibration were elaborated.

All nodules were rapidly and highly enhanced compared with the edematous spinal cord, along with feeding arteries and draining veins (Fig. 3). CEUS showed that cyst capsules and altered spinal cord between the nodules did not present enhancement, discordantly from MRI findings (Figs. 3 and 4). On the other hand, a nodular lesion at C-7, which protruded on the medullar surface, showed a rapid and homogeneous enhancement with a late washout. A vascular peduncle was clearly identifiable. All these findings were in favor of hemangioblastoma. Caudally to this nodule, at the T3-T4 level, the medulla appeared edematous and hyperechoic; however, no superficial lesion was identifiable. Again, CEUS showed the presence of a deep-seated intramedullary nodule, with ultrasonographic characteristics similar to those of the first nodule (Fig. 5). With a delicate dissection along the spinal cord midline, we started to coagulate afferent vessels, and this lesion was also removed. Finally, a nodular lesion at the level of the right C-5 root was identified and removed.

All the tumors were completely removed with a microneurosurgical technique. The D-wave did not show any change with respect to the basal level. Laminae removed were then replaced and secured to the surrounding ver-

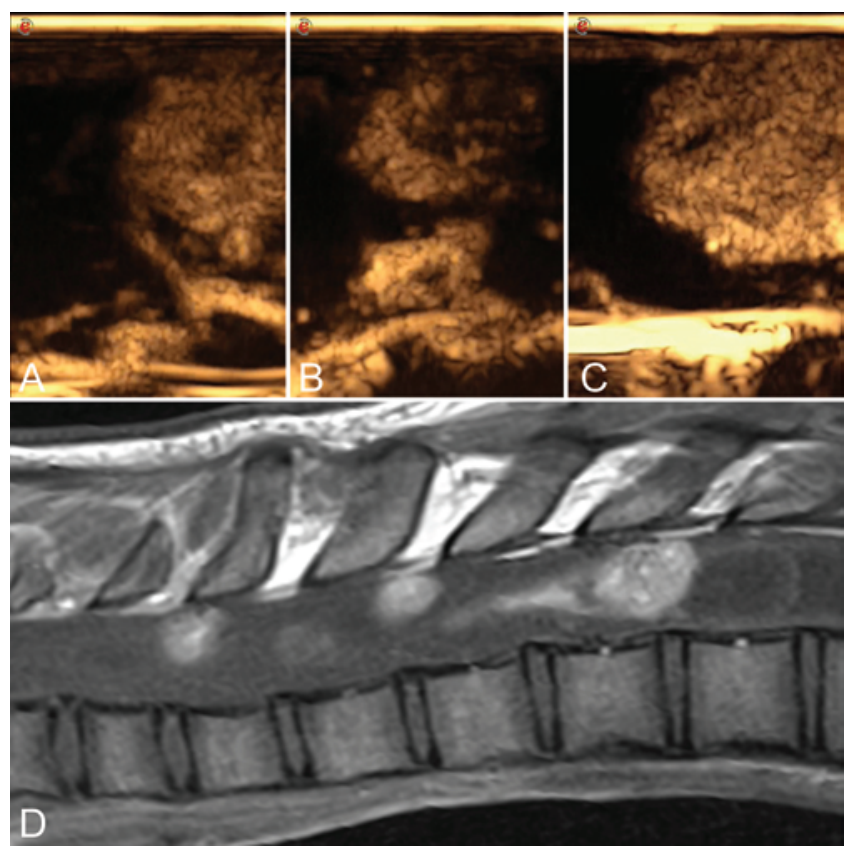

FIG. 3. Intraoperative CEUS visualization of 3 nodular lesions (A-C) compared with the corresponding preoperative post-Gd MRI scan visualization (D). 


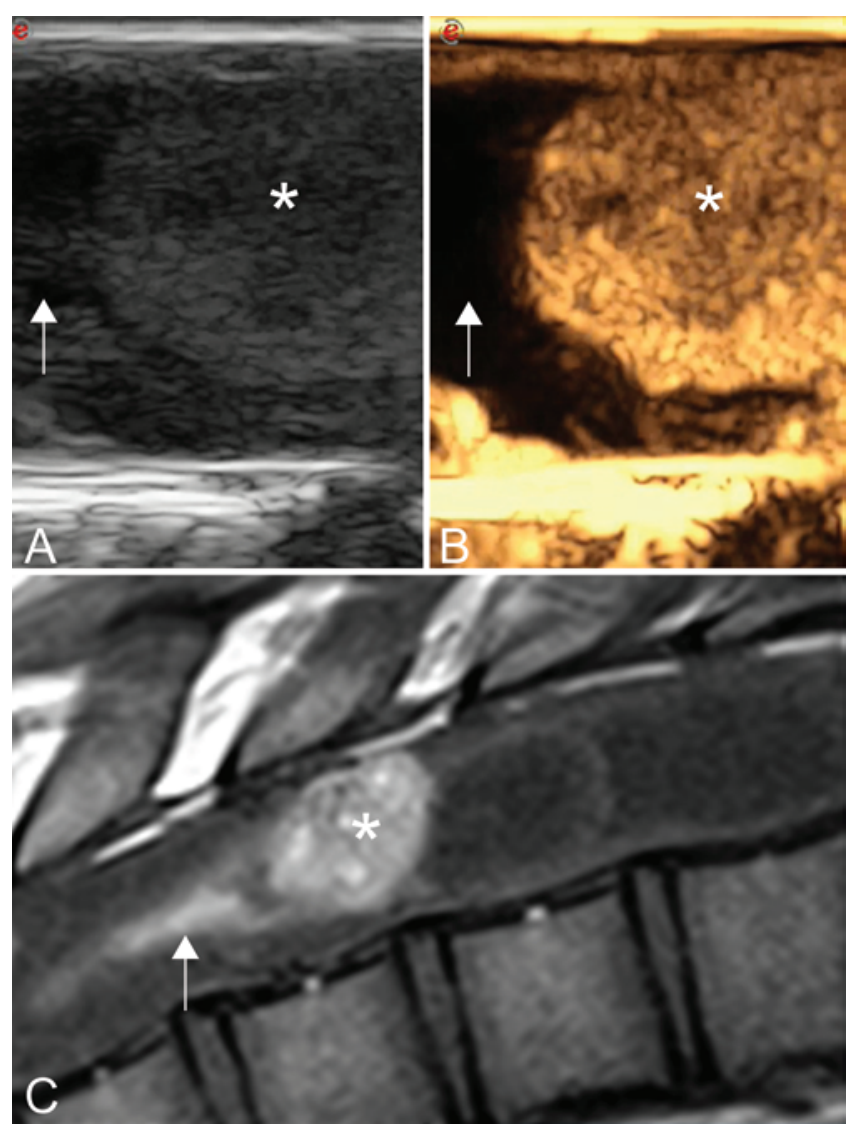

FIG. 4. A: Both the lesion (asterisk) and the spinal cord (arrow) appear hyperechoic at B-mode examination. B: The contrast enhancement of the lesion (asterisks) is clearly visible, with a dotted homogeneous pattern, while the spinal cord (arrow) is not enhancing. These findings are different from those of the preoperative MRI scans (C), in which the spinal cord (arrow) is also enhanced after $\mathrm{Gd}$ administration, giving the impression of an infiltrative diffuse lesion.

tebral bone with titanium plates and self-tapping screws (Fig. 6).

The histological diagnosis confirmed the intraoperative ultrasonographic findings, and it was in favor of hemangioblastomas. The patient had an uneventful postoperative course. Two months after surgery he fully recovered
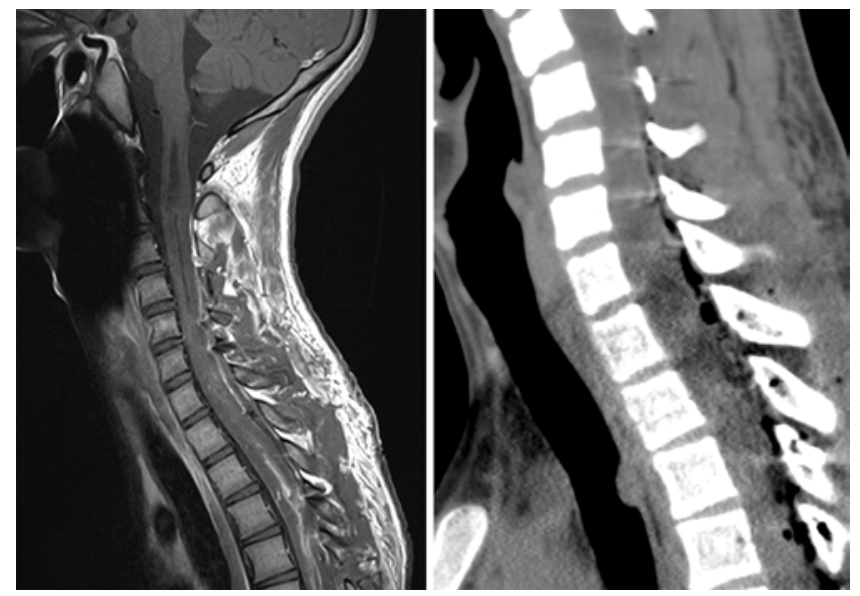

FIG. 6. Left: Postoperative MRI showing removal of tumors. Initial reduction of the cervical syrinx is visible. Right: CT scans depicting reposition of laminae.

his motor symptoms, and only a mild hypoesthesia of the right hand was still present.

\section{Discussion}

Hemangioblastomas usually have a very discrete tumor-spinal cord interface and do not infiltrate the spinal cord substance. The resection of the lesion also usually determines syrinx improvement; no syrinx cavity drainage is necessary. ${ }^{5,14,17,29}$ Recurrence is in most cases due to incomplete resection; therefore, accurate surgical planning is mandatory. MRI represents the gold standard for the diagnosis of intradural tumors; however, as shown in this case, MRI findings can sometimes be misinterpreted. In fact, the spinal cord at the C2-T2 level appeared enlarged, with altered signal. Cyst capsules showed intense enhancement after Gd injection on T1-weighted images, appearing as nodular lesions, and were therefore considered as cystic degeneration of the solid nodular components, not as tumor-associated syringomielic cavities.

Only the ultrasonographic examination, in particular after UCA administration, showed actual findings: B-mode US delineated 3 distinct hyperechoic nodules within an otherwise hyperechoic spinal cord. iCEUS

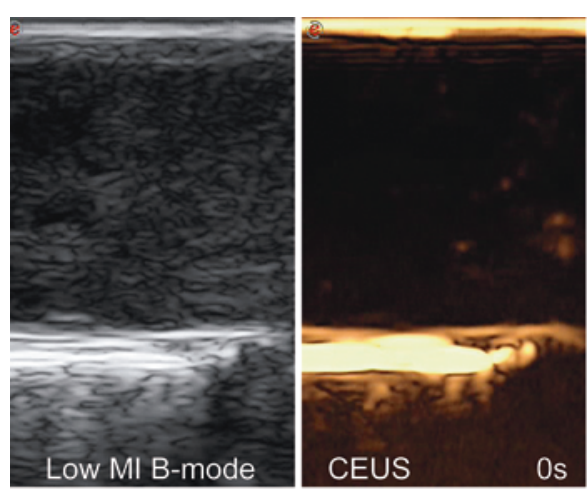

Baseline

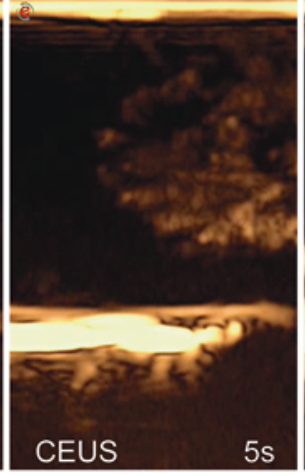

Arterial phase

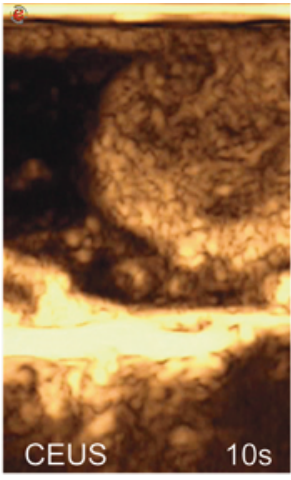

Peak

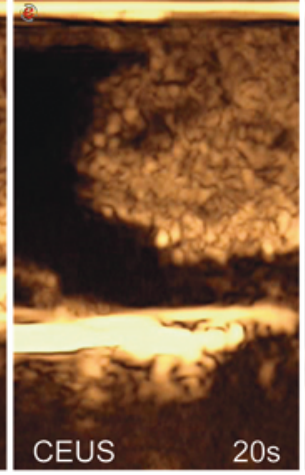

Parenchymal phase

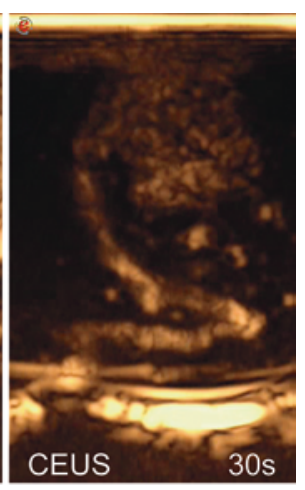

Venous phase

FIG. 5. Time frame of UCA diffusion in the T3-T4 hemangioblastoma. The contrast medium allows rapid visualization of the lesion, reaching a peak of enhancement after 10 seconds from intravenous injection. $\mathrm{MI}=$ mechanical index; $\mathrm{s}=$ seconds. 
clearly highlighted the 3 nodules along with their feeding and draining vessels while not enhancing the spinal cord, particularly in the areas where the MRI was misleading, showing Gd enhancement. This difference might be explained by the different nature of the 2 contrast agents: UCAs are purely intravascular while MRI paramagnetic contrast agents are vascular and interstitial. The post-Gd enhancement at MRI of the perilesional cysts and edematous spinal cord could be explained by the increased intramedullary venous pressure due to the high-flow lesions that caused a local venous congestion with possible extravasation and diffusion in the interstitial compartment, while UCA, being the size of a red blood cell, remained within the vascular spaces.

In spinal tumor US-guided surgery, the B-mode examination is usually sufficient to depict the lesions prior to dural opening. , $^{2,12,13,26,28,33}$ However, in certain cases the use of other US techniques as adjuncts could be helpful in better characterizing the nature of the lesions, in particular if they present as intramedullary.

Intraoperative US with color Doppler can be used to visualize an encased tumor or displaced vessels and the origin of the vascular supply to the main tumor. It facilitates the identification of important neurovascular structures during tumor removal but nonetheless presents certain limitations: low-flow veins are not always visible and small vessels may be overestimated due to blooming artifacts. ${ }^{9}$ Furthermore, it may be difficult to follow displaced vessels with a proper insonation angle because they do not have a linear course and also because of the limit imposed by the margins of bone removal, which reduces the accuracy of the Doppler signal. ${ }^{9}$

Over the years, a number of studies regarding UCA application in liver and many other organs have been performed. ${ }^{15,25}$ On the other hand, the intraoperative application of CEUS in neurosurgery is still limited. As already established for brain tumor surgery, iCEUS is a valuable tool to better highlight cerebral lesions and obtain additional information regarding their vascular and perfusion patterns.

Relying on our experience with iCEUS in brain tumor surgery, ${ }^{22,23}$ we decided to use this method in this case. CEUS allowed us to further highlight the lesion and differentiate it from an infiltrative spinal cord tumor like astrocytoma. In particular, the polar cystic areas and spinal cord did not show contrast enhancement, differently from MRI findings. On the other hand, the nodular Doppler and CEUS characteristics were in favor of a noninfiltrative lesion, like hemangioblastoma.

One possible limitation of the CEUS technique is that the analysis could be performed only by analyzing one portion of the lesion at a time. It is consequently mandatory to accurately scan the lesion in B-mode and color Doppler imaging before performing iCEUS. This is necessary to evaluate the more significant portion of the lesion and to obtain as much information as possible in regard to the timing. As demonstrated in our case, a correlation between iCEUS and preoperative MRI (T1-weighted Gd) presents some major obstacles when comparing the degree of enhancement after contrast between the techniques. In fact, microbubbles are purely intravascular, whereas MRI con- trast agent is mainly interstitial. Furthermore, CEUS is a dynamic perfusion imaging, and MRI is static.

All ioUS findings were very useful in approaching this lesion, and iCEUS offered further insight, highlighting the lesion and describing its perfusion pattern. This led to a change of surgical strategy and helped us to achieve total resection of the tumors.

In our experience, iCEUS has proven to be a simple and relatively inexpensive technique representing a real-time dynamic procedure that can be performed during spinal tumor surgery. The ability of iCEUS to highlight vascular structures made it easier for the surgeon to identify the vascular peduncles, giving further insight into the surgical strategy and facilitating vascular deafferentation of the lesion and then its surgical removal.

\section{References}

1. Baker KB, Moran CJ, Wippold FJ II, Smirniotopoulos JG, Rodriguez FJ, Meyers SP, et al: MR imaging of spinal hemangioblastoma. AJR Am J Roentgenol 174:377-382, 2000

2. Chadduck WM, Flanigan S: Intraoperative ultrasound for spinal lesions. Neurosurgery 16:477-483, 1985

3. Chu BC, Terae S, Hida K, Furukawa M, Abe S, Miyasaka $\mathrm{K}$ : MR findings in spinal hemangioblastoma: correlation with symptoms and with angiographic and surgical findings. AJNR Am J Neuroradiol 22:206-217, 2001

4. Conway JE, Chou D, Clatterbuck RE, Brem H, Long DM, Rigamonti D: Hemangioblastomas of the central nervous system in von Hippel-Lindau syndrome and sporadic disease. Neurosurgery 48:55-63, 2001

5. Cristante L, Herrmann HD: Surgical management of intramedullary hemangioblastoma of the spinal cord. Acta Neurochir (Wien) 141:333-340, 1999

6. Cui DM, Che XM, Xu QW, Gu SX, Shi W, Yang F: [Diagnosis and treatment of spinal cord hemangioblastoma.] Zhonghua Yi Xue Za Zhi 87:308-310, 2007 (Chinese)

7. Duong LM, McCarthy BJ, McLendon RE, Dolecek TA, Kruchko C, Douglas LL, et al: Descriptive epidemiology of malignant and nonmalignant primary spinal cord, spinal meninges, and cauda equina tumors, United States, 2004-2007. Cancer 118:4220-4227, 2012

8. Epstein FJ, Farmer JP, Schneider SJ: Intraoperative ultrasonography: an important surgical adjunct for intramedullary tumors. J Neurosurg 74:729-733, 1991

9. Gibbs V, Cole D, Sassano A: Ultrasound Physics and Technology: How, Why and When. London: Churchill Livingstone/Elsevier, 2009

10. Kaffenberger DA, Shah CP, Murtagh FR, Wilson C, Silbiger ML: MR imaging of spinal cord hemangioblastoma associated with syringomyelia. J Comput Assist Tomogr 12:495498, 1988

11. Lee DK, Choe WJ, Chung CK, Kim HJ: Spinal cord hemangioblastoma: surgical strategy and clinical outcome. J Neurooncol 61:27-34, 2003

12. Lerch K, Völk M, Neumann C, Nerlich M: [Intraoperative spinal sonography.] Ultraschall Med 23:113-118, 2002 (Ger)

13. Maiuri F, Iaconetta G, Gallicchio B, Stella L: Intraoperative sonography for spinal tumors. Correlations with MR findings and surgery. J Neurosurg Sci 44:115-122, 2000

14. Mandigo CE, Ogden AT, Angevine PD, McCormick PC: Operative management of spinal hemangioblastoma. Neurosurgery 65:1166-1177, 2009

15. Martegani A, Aiani L, Borghi C: The use of contrastenhanced ultrasound in large vessels. Eur Radiol 14 (Suppl 8):73-86, 2004

16. McCormick PC, Stein BM: Intramedullary tumors in adults. Neurosurg Clin N Am 1:609-630, 1990 
17. Murota T, Symon L: Surgical management of hemangioblastoma of the spinal cord: a report of 18 cases. Neurosurgery 25:699-708, 1989

18. Oppenlander ME, Spetzler RF: Advances in spinal hemangioblastoma surgery. World Neurosurg 74:116-117, 2010

19. Pai SB, Krishna KN: Secondary holocord syringomyelia with spinal hemangioblastoma: a report of two cases. Neurol India 51:67-68, 2003

20. Piscaglia F, Bolondi L: The safety of Sonovue in abdominal applications: retrospective analysis of 23188 investigations. Ultrasound Med Biol 32:1369-1375, 2006

21. Piscaglia F, Nolsøe C, Dietrich CF, Cosgrove DO, Gilja OH, Bachmann Nielsen M, et al: The EFSUMB Guidelines and Recommendations on the Clinical Practice of Contrast Enhanced Ultrasound (CEUS): update 2011 on non-hepatic applications. Ultraschall Med 33:33-59, 2012

22. Prada F, Mattei L, Del Bene M, Aiani L, Saini M, Casali C, et al: Intraoperative cerebral glioma characterization with contrast enhanced ultrasound. BioMed Res Int 2014:484261, 2014

23. Prada F, Perin A, Martegani A, Aiani L, Solbiati L, Lamperti $\mathrm{M}$, et al: Intraoperative contrast-enhanced ultrasound for brain tumor surgery. Neurosurgery 74:542-552, 2014

24. Prada F, Vetrano IG, Filippini A, Del Bene M, Perin A, Casali C, et al: Intraoperative ultrasound in spinal tumor surgery. J Ultrasound 17:195-202, 2014

25. Quaia E, Calliada F, Bertolotto M, Rossi S, Garioni L, Rosa $\mathrm{L}$, et al: Characterization of focal liver lesions with contrastspecific US modes and a sulfur hexafluoride-filled microbubble contrast agent: diagnostic performance and confidence. Radiology 232:420-430, 2004

26. Rasmussen IA Jr, Lindseth F, Rygh OM, Berntsen EM, Selbekk T, Xu J, et al: Functional neuronavigation combined with intra-operative 3D ultrasound: initial experiences during surgical resections close to eloquent brain areas and future directions in automatic brain shift compensation of preoperative data. Acta Neurochir (Wien) 149:365-378, 2007

27. Rebner M, Gebarski SS: Magnetic resonance imaging of spinal-cord hemangioblastoma. AJNR Am J Neuroradiol 6:287-289, 1985

28. Regelsberger J, Fritzsche E, Langer N, Westphal M: Intraoperative sonography of intra- and extramedullary tumors. Ultrasound Med Biol 31:593-598, 2005

29. Samii M, Klekamp J: Surgical results of 100 intramedullary tumors in relation to accompanying syringomyelia. Neurosurgery 35:865-873, 1994

30. Schellinger KA, Propp JM, Villano JL, McCarthy BJ: Descriptive epidemiology of primary spinal cord tumors. J Neurooncol 87:173-179, 2008

31. Spetzger U, Bertalanffy H, Huffmann B, Mayfrank L, Reul J, Gilsbach JM: Hemangioblastomas of the spinal cord and the brainstem: diagnostic and therapeutic features. Neurosurg Rev 19:147-151, 1996

32. Xu QW, Bao WM, Mao RL, Yang GY: Magnetic resonance imaging and microsurgical treatment of intramedullary hemangioblastoma of the spinal cord. Neurosurgery 35:671676, 1994

33. Zhou H, Miller D, Schulte DM, Benes L, Bozinov O, Sure $\mathrm{U}$, et al: Intraoperative ultrasound assistance in treatment of intradural spinal tumours. Clin Neurol Neurosurg 113:531537,2011

\section{Author Contributions}

Conception and design: Prada, Vetrano. Acquisition of data: Prada, Vetrano, Valentini. Analysis and interpretation of data: Prada, Vetrano, Valentini. Drafting the article: Prada, Vetrano, Nataloni, Del Bene. Critically revising the article: all authors. Reviewed submitted version of manuscript: all authors. Approved the final version of the manuscript on behalf of all authors: Prada. Study supervision: DiMeco.

\section{Correspondence}

Francesco Prada, Department of Neurosurgery, Fondazione IRCCS “Istituto Neurologico Carlo Besta," Via G. Celoria 11, Milan 20133, Italy. email: francesco.prada@istituto-besta.it. 